\title{
3D acquisition of mirroring objects using striped patterns
}

\author{
Marco Tarini *, Hendrik P.A. Lensch, Michael Goesele, \\ Hans-Peter Seidel
}

Max-Planck-Institut für Informatik, Saarbrücken, Germany

Received 11 July 2002; received in revised form 16 August 2004; accepted 8 November 2004

Available online 16 February 2005

\begin{abstract}
Objects with mirroring optical characteristics are left out of the scope of most 3D scanning methods. We present here a new automatic acquisition approach, shape-from-distortion, that focuses on that category of objects, requires only a still camera and a color monitor, and produces range scans (plus a normal and a reflectance map) of the target. Our technique consists of two steps: first, an improved environment matte is captured for the mirroring object, using the interference of patterns with different frequencies to obtain sub-pixel accuracy. Then, the matte is converted into a normal and a depth map by exploiting the self-coherence of a surface when integrating the normal map along different paths. The results show very high accuracy, capturing even smallest surface details. The acquired depth maps can be further processed using standard techniques to produce a complete 3D mesh of the object.

(c) 2004 Elsevier Inc. All rights reserved.
\end{abstract}

PACS: $07.05 . \mathrm{H} ;$ 42.79.B; 07.05.K

Keywords: 3D Scanning; Mirroring surfaces; Shape from shading; Shape from distortion

\footnotetext{
* Corresponding author.

E-mail address: tarini@isti.cnr.it (M. Tarini).
}

1524-0703/\$ - see front matter (c) 2004 Elsevier Inc. All rights reserved.

doi:10.1016/j.gmod.2004.11.002 


\section{Introduction}

In recent years, research on $3 \mathrm{D}$ acquisition led to the development of working techniques capable of producing digital 3D models of real world objects. These techniques differ in many aspects, including precision, scanning time, amount of required human interaction, economic affordability, type of resulting models, captured attributes, and so on. Another central aspect are the categories of objects that can be scanned by the specific techniques, i.e., which constraints limit the possible targets in terms of size, weight, complexity, and surface properties.

In particular, theory and practice show that objects with mirroring surface properties are outside the scope of almost all current standard approaches for high quality acquisition. In all active scanning methods (e.g., laser scanning, structured light, and time of flight - see [1]) the problem is that the active signal (e.g., projected pattern or laser) is mainly reflected along the specular direction so that almost no light reaches the sensor. In addition, specular reflections of an object are effected by the surface normal and can therefore lead to the effect that the projected pattern is perceived on the wrong position [2]. Shape from shading based approaches [3] require the surface to be Lambertian and do not work for glossy, especially not for mirroring surfaces. In shape from silhouette approaches [4], beside of the general problem of lacking precision, the silhouettes of mirroring objects are hard to detect automatically since the whole environment is reflected in the object.

However, mirroring objects may nevertheless be scanned by the approaches mentioned above if they are previously painted (or covered with white dust) to approximate Lambertian surface behavior. But this is clearly invasive and unpractical for many applications.

All in all, there is an urgent need to use optical techniques to measure the shape of an object with a specular surface, especially technical components, pieces of art or even everyday objects - like knifes, forks or keys - without changing their optical properties.

In this article we present a novel approach, shape from distortion, to reconstruct the surface of a mirroring object with high precision. It uses only standard, cheap hardware (a monitor and a camera), and care has been taken to achieve relatively short acquisition times. Our approach consists of two steps:

- an improved environment matte extraction that uses the interference of patterns of different frequencies to obtain sub-pixel accuracy relative to the projected pattern,

- followed by a step that converts the matte into a normal and a depth map by exploiting the self coherence of a surface when integrating the normal map along different paths.

The process is very modular: even if the second step requires the output of the first one as input, each can also be used separately in different contexts.

Before giving a more detailed overview over our technique in Section 3 we will briefly summarize related work by other researchers on the subject of specular objects scanning. 


\section{Previous work on mirroring object acquisition}

Several research groups developed variations of standard range scanning techniques that are capable of scanning specular objects.

In Oren and Nayar [5], 3D surface profiles of specular objects are generated by observing the movements of virtual (i.e., reflected) features on their surface. Similarly, Zheng et al. [6] rotated the specular object on a turntable under the illumination of two linear fluorescent lamps, and estimating normal and geometry by the motion of the reflection during the rotation. Later, Zheng and Murata [7] extended this approach, using circular-shaped light sources, so that, if there are no occlusions due to the object shape, a highlight can be observed on all surface points regardless of their normal, thus increasing the number of possible shapes that can be recovered. While they can derive the complete geometry of an object in a single scan, the accuracy of the generated models is limited and special hardware is required.

The reconstruction of shape and diffuse or glossy BRDF from images of the objects illuminated by a point light at different positions has been investigated by Belhumeur and Kriegman [8]. In that approach the BRDF of the object is an unknown to be recovered as well as its shape, leading to a more complex problem that consequently requires hundreds of images to be solved. More importantly, their approach cannot provide the angular resolution that is required for measuring surfaces with a pure mirroring BRDF.

In [2], a laser scanning system is proposed where a shield mask limits the direction of incident light. The prototype system is able to scan specular as well as Lambertian surfaces with high accuracy but at a very low speed since each position on the surface has to be seen under various angles (100 measurement points in $20 \mathrm{~min}$ [9]).

The quality of flat or nearly flat surfaces such as a die or sheet metal has often been measured by observing the reflection of regular grids (see, for example [1] for an overview). Similar to reflection lines used in geometric modelling [10], the accuracy of these methods is high because measured surface normals are used to reconstruct the shape of the object. A similar method has also been proposed by [11] to measure the shape of the human cornea. Our method also benefits from this measurement principle in terms of accuracy and extends it to recover dense range maps of arbitrary shaped objects.

Park and Cho [12] place a specular object in a retroreflective hemisphere and illuminate its surface with a laser beam. The reflected beam is measured and the position on the object where it was hit by the retroreflected beam is calculated. The next beam is directed at this surface point under a different angle. Once the position and corresponding normal for one surface point is known, the shape and surface normals of a sufficiently smooth object can be reconstructed recursively. The main problem of this approach is that errors are accumulated in each step and not corrected. With growing distance from the initial point the data become more and more unreliable.

In their recent work on this subject, Savarese and Perona $[13,14]$ use a $2 \mathrm{D}$ pattern consisting of points identified by the intersection of three coplanar lines, and manage to recover from their reflection some attributes of the mirroring surface, i.e., relationships between depth and normal. This can be used to locally reconstruct the surface 
itself, if an approximation of its distance is provided, or some a priori knowledge of the shape of the mirror (a sphere or a flat mirror) is known. Still, the detection and labelling of elements of the patterns is far from automatic and indeed quite problematic for not trivially shaped mirror. More important, the measurements are very sparse. Typically their number is around a dozen, so no assumption of point-to-point continuity is possible. Therefore, the technique can be useful as auxiliary rather that substitute for typical range scanning technique.

Following a totally different approach, Bonfort and Sturm [15] showed that the standard voxel carving techniques can be adapted to the case of mirroring objects. The mirroring object is photographed in front of a known surrounding environment from different point of views. The voxels are then detected not to be on the surface of the target object - and thus discarded - whenever it is not possible to coherently associate a normal to it, that is compatible with the perceived reflection. The mirroring object surface is reconstructed as the set of surviving voxels. This technique inherits the characteristic of other voxel carving approaches: while they can be useful to recover a rough approximation of the global shape of the capture object, the achieved resolution and accuracy are typically very low. In this sense, this technique is complementary to the one that we are presenting here: the latter on the contrary produces range maps that are a very accurate, but partial, representations of the targeted surface (multiple range maps are typically necessary to reconstruct an entire model).

Finally, a technique that aims at capturing more the appearance of mirroring objects rather than their 3D geometry has been presented by Zongker et al. [16]. They introduced environment mattes which capture how light impinging from the environment is reflected by the object. We base our acquisition technique on environment matting and extend this approach. Environment mattes will be revisited and explained in more detail in Section 4.

\section{Overview}

To obtain the 3D geometry, we observe images of a nearby monitor that are reflected in the mirroring object. The shape of the object is recovered by investigating the way the perceived reflection of the monitor is distorted. We call this process shape from distortion.

To represent the distortion we use an environment matte $[16,17]$ which is a general way to model the optical interactions between an object and its surroundings. Our system consists of, first, capturing a matte, and then using it to produce a range map representing the surface of the investigated object. The next two sections are dedicated to one phase each:

Section 4 describes a new automatic way to capture the environment matte with high accuracy: we capture five images of the object while it reflects repetitive patterns of different frequencies displayed on a nearby monitor (Fig. 1A). These images (Fig. 1B) are used to construct the matte (Fig. 1C) with sub-pixel precision, i.e., for each pixel in the captured images we compute the exact $2 \mathrm{D}$ position of the point where the reflected ray hits the monitor. 


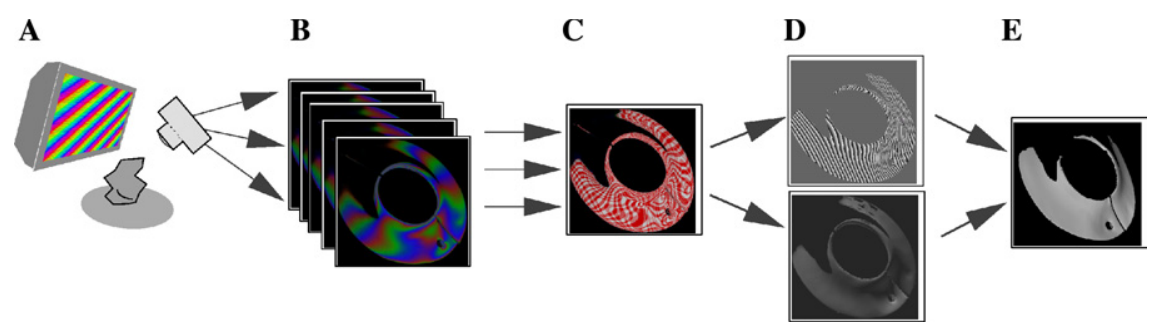

Fig. 1. The big picture of shape from distortion: the target object is photographed (A) while reflecting some pattern displayed by a monitor. The captured images are used (B) to compute an environment matte (C) from which we extract a normal map together with a depth map (D) that are embedded into the final 3D range scan $(\mathrm{E})$.

In Section 5, we describe how the geometry of the object is reconstructed in the form of a depth and a normal map (Fig. 1D) from the resulting matte. Given the position of the monitor and a tentative normal at each pixel, the environment matte relates the normals to corresponding depth values, and vice versa. Since both are initially unknown we apply another constraint to find their absolute values: we minimize the incoherence of the surface that occurs integrating the normal map along different paths.

The final output of the presented algorithm is a standard range image, a 2D data structure that for each point stores the distance of the scanned object along the corresponding ray (Fig. 1D) enriched by a normal map, plus a reflectance map derived directly from the matte. A complete model of the object can be constructed out of multiple range maps using well known techniques for scan alignment [18,19], merging $[20,21]$, and surface simplification [22].

The described steps of our approach are in practice preceded by a calibration procedure which is explained in Section 6. We need to recover the response of the camera to the intensity of the monitor with respect to the three color channels, since colored stripe patterns are applied during scanning. Further, a calibration of the intrinsic camera parameters is performed, and the relative monitor position is measured. This calibration has to be done only once and can be reused for subsequent scans as long as the monitor and the camera are not moved.

Finally, in Section 7 we show some experimental results and in Section 8 we conclude and give some directions for future work.

\section{Matting extraction}

In this section, we describe an algorithm to acquire an Environment Matte that captures with sub-pixel precision the way a given mirroring object reflects its environment.

\subsection{Previous matting techniques}

Matting is a category of $2 \mathrm{D}$ techniques that consists, in the general case, in capturing how the environment (in most cases a far background) is filtered, reflected, 
occluded, etc., by a target object (given a fixed illumination and viewpoint). This information is then typically used to synthesize images where the same foreground object is realistically shown in a different, possibly synthetic, environment.

The most simple matte, known as blue (or, more recently, green) screen matte [23], records for each pixel a real value in the interval $[0 \ldots 1]$ modelling how much of the background is visible at that pixel. This technique is widely used in cinematography to replace the background of an arbitrary scene. The information is captured at each frame showing the object in front of a homogeneous blue, yellow, or green background.

More information is recovered by an environment matte [16,17], which stores, for each pixel position $(u, v)$, the texture coordinate $\vec{o}(u, v)$ over an environment texture of the texel that, possibly after a reflection or refraction with the object, is seen at the position $(u, v)$. The object can then be placed into arbitrary backgrounds while maintaining its reflection characteristics. The original environment matte does not store the coordinate of a single point but the rectangular area of the environment texture that contributes to the considered pixel; however, for our purposes (mirroring surface reconstruction) we need to use only use main reflection direction.

Note that the environment matte usually refers to a background placed behind the object, and visible through it, while for our purposes we need the matte to refer rather to the environment in front of the object, and reflected by it. However, the mentioned matting techniques are not limited to recover background environment mattes and would work just as well for foreground environment mattes.

In [16] Zongker et al. extracted the necessary information $\vec{o}(u, v)$ from a series of images of reflected binary encoded stripes displayed by a monitor that is placed at the position of the virtual texture. Here, the frequency of the pattern is doubled from frame to frame using $1,2,4, \ldots, 2^{n}$ stripes. The low frequency patterns determine a rough approximation of the location of $\vec{o}(u, v)$, while higher frequency ones provide a more and more accurate refinement of that estimate. Similar approaches of spatiotemporal modulation of the illumination are commonly used in other 3D scanning applications, e.g. [24]. Unfortunately, in current approaches the accuracy of the system is limited by the resolution of the projected pattern with the highest frequency. Furthermore, little information is obtained from the images with lower frequencies which only roughly determine the position.

The environment matting technique has further been extended in two ways by Chuang et al. [17]: the first one increases the quality. It assumes that a pixel value at $(u, v)$ results from an area of the texture weighted by a Gaussian rather than a rectangular region. A stripe with Gaussian profile is swept smoothly over the monitor. The stream of 100-300 frames is recorded by a video camera to obtain the position $\vec{o}(u, v)$ and the spatial extend of the Gaussian filter that has to be applied.

Another improvement reduces the effort of capturing the matte to just a single frame, allowing real-time mattes. However, it only works for background textures. The binary stripe pattern is replaced by smoothly varying transition of pure red, green, and blue color, a planar slice through the RGB cube. The resulting matte consists of the texture coordinates $\vec{o}(u, v)$ and an opacity value $\alpha(u, v)$. While the acqui- 
sition is considerably accelerated it also introduces a lot of noise. To improve quality, the recovered matte is filtered by combining neighboring pixels which limits the accuracy.

\subsection{Our matte}

In our approach, the matte is defined by the following attributes at each pixel $(u, v)$ :

- the 2D position $\vec{o}(u, v)$ where the ray through pixel $(u, v)$, reflected by the object, intersects the monitor. $\vec{o}(u, v)$ will be recovered with sub-pixel precision with respect to the resolution of the monitor which is required for accurate $3 \mathrm{D}$ reconstruction.

- the reflectance $\alpha(u, v)$ as a color vector, encoding the perceived attenuation of light emitted by the monitor. For a perfect mirror $\alpha(u, v)$ will be $(1,1,1)^{\mathrm{T}}$ while for points outside the silhouette of the object it will be $(0,0,0)^{\mathrm{T}}$.

Regarding the content, our matte is very similar to the real-time captured matte [17]. However, our application scenario demands a much higher accuracy so that a single image is not sufficient to recover a high quality matte. Even more important, we have to avoid filtering and want to be able to reconstruct the value of $\vec{o}(u, v)$ for each pixel of the captured image totally independent on the values of neighboring pixels. This is because the origin of a reflected ray may change drastically for relatively small variations of the surface normal. Combining values from neighboring pixels thus would introduce too large errors. We also want to keep the number of required images as low as possible.

The new pattern scheme we apply for the matte extraction consists of four images. The patterns $M_{1 \ldots 4}$ (visible in Fig. 2) are striped along different directions. After calibration (see Section 6) the repeated period of the stripes is perceived by the camera as the periodic function shown in Fig 3: the signal is built out of hat functions for the RGB color channels displaced in such a way that they always sum up to one.

For each location $(u, v)$, the quadruple of observed color values $c_{i}, i \in[1 \ldots 4]$ for the four patterns is sufficient to determine $\vec{o}(u, v)$ robustly and with sub-pixel precision: we first determine the position (or phase) $x_{1 \ldots 4}$ inside one stripe for each of the four patterns; using the interference patterns we then recover the number of the stripe $y_{1 \ldots 4}$ in which the point is located.
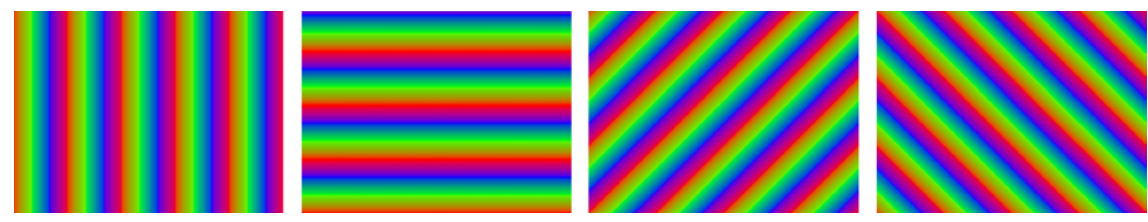

Fig. 2. Patterns $M_{1 \ldots 4}$ sent to the monitor to capture the reflected environment matte. 


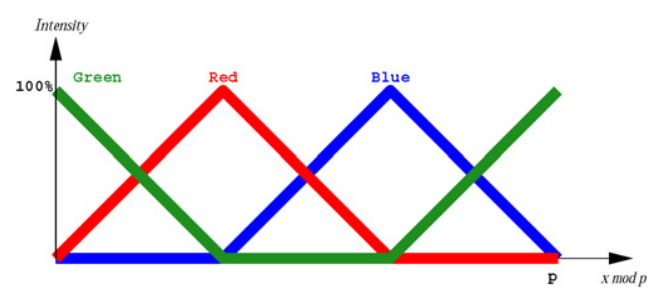

Fig. 3. Each striped pattern shown in Fig. 2 is perceived in the camera as a repetition of the following values of red, green, and blue (up to a scale). (For interpretation of the references to color in this figure legend, the reader is referred to the web version of this paper.)

A perceived color pixel $c_{i}$ identifies uniquely $x_{i}$ as the abscissa, inside the function presented in Fig. 3, that best matches the RGB color values of $c_{i}$. We find $x_{i}$ using the following algorithm:

(1) The three color values are sorted yielding $c_{i}[\min ] \leqslant c_{i}[\operatorname{med}] \leqslant c_{i}[\max ](\operatorname{mini}-$ mum, median, maximum).

(2) The order of the color channels locates $x_{i}$ inside one of the six regions, where each region is delimited by the intersection of the $r, g$, and $b$ hat functions of Fig. 3. Let regionNr $\in\{1 \ldots 6\}$ be the index of that region.

(3) The exact position of $x_{i}^{\prime} \in[0 \ldots 1)$ within the region is found by

$$
x_{i}^{\prime}=\frac{2 \cdot\left(c_{i}[\operatorname{med}]-c_{i}[\min ]\right)}{\left(c_{i}[\max ]-c_{i}[\min ]\right)+\left(c_{i}[\operatorname{med}]-c_{i}[\min ]\right)} .
$$

Considering the magnitude of $c_{i}$ [med] with respect to the minimal and maximal values takes into account darkening due to absorbed light. Additionally, it also works correctly if the minimum value is not zero. This reduces errors introduced by ambient lighting and color shifts. In an ideal case, the minimum value would always be zero by construction of the signal.

(4) If the in the region regionNr the median function is decreasing, that is if regi onNr is an odd number, then the value $x_{i}^{\prime}$ must be reversed, with $x_{i}^{\prime} \leftarrow 1-x_{i}^{\prime}$.

(5) The final $x_{i}$ is given by the value of $x_{i}^{\prime}$ scaled and biased to take into account the region it belongs to

$$
x_{i}=\frac{\text { regionNr }-1}{6}+\frac{x_{i}^{\prime}}{6} \text {. }
$$

Repeating this algorithm for each color $c_{1 \ldots 4}$ one ends up with four values $x_{1 \ldots 4}$, each value $x_{i}$ identifying where, inside a still unknown stripe $y_{i}$, the origin $\vec{o}(u, v)$ of the monitor point reflected to pixel $(u, v)$ is located. The unknowns $y_{i}$ are found by exploiting the interference of the four patterns.

In fact, the two diagonal patterns $M_{3}$ and $M_{4}$ have a different period for the stripes than $M_{1}$ and $M_{2}$, e.g., each row of $M_{1}$ has five repetitions while each row of $M_{3}$ has four. Since four and five are coprimes, there is only one possible solution $y_{1 \ldots 4}$ that justifies the measured values $x_{1 \ldots 4}$. To find it we need to solve a system with an equation for each striped pattern used: 


$$
\begin{aligned}
o_{x} & =\left(x_{1}+y_{1}\right) / 5, \\
o_{y} & =\left(x_{2}+y_{2}\right) / 5, \\
o_{x}+o_{y} & =\left(x_{3}+y_{3}\right) / 4, \\
o_{x}-o_{y} & =\left(x_{4}+y_{4}\right) / 4,
\end{aligned}
$$

where $\vec{o}(u, v)=\left(o_{x}, o_{y}\right)$. The unknowns are $o_{x}, o_{y}$, and the integer values $y_{1 \ldots .}$, with $y_{1,2} \in 0, \ldots, 4$ and $y_{3,4} \in 0, \ldots, 3$. Note that we have an occurrence of the shown system for each position $(u, v)$ on the matte, and each one is solved separately. The system is solved by:

$$
\begin{aligned}
& 4 y_{1}-5\left(y_{3}+y_{4}\right)=4 x_{1}-5 x_{3}+5 x_{4}, \\
& 4 y_{2}-5\left(y_{3}-y_{4}\right)=4 x_{2}-5 x_{3}-5 x_{4} .
\end{aligned}
$$

Since the left-hand sides of both equations represent integer values, we round the values of the two right-hand sides, that are knowns, to their closest integer values $i_{1}$ and $i_{2}$, respectively (the magnitude of the rounding can be used as a measure of our confidence for the value of $\vec{o}$ at that position). What remains to solve is:

$$
\begin{aligned}
& 4 y_{1}-5 d_{1}=i_{1}, \\
& 4 y_{2}-5 d_{2}=i_{2},
\end{aligned}
$$

where $d_{1}$ and $d_{2}$ are the sum and the difference between $y_{3}$ and $y_{4}$, i.e., $d_{1}=\left(y_{3}+y_{4}\right)$ and $d_{2}=\left(y_{3}-y_{4}\right)$. Eq. (5) are two simple Diophantic equations to be solved separately yielding:

$$
\begin{aligned}
& \left(y_{1}, d_{1}\right)=i_{1}(3,4)+k_{1}(5,4), \\
& \left(y_{2}, d_{2}\right)=i_{2}(3,4)+k_{2}(5,4),
\end{aligned}
$$

for any $k_{1,2} \in \mathrm{IN}$. We chose for $k_{1}$ (and $k_{2}$ ) the only value that puts $y_{1}$ (and $y_{2}$ ) in the required interval $[0 \ldots 4]$.

With the values $d_{1}$ and $d_{2}$ we trivially compute values for $y_{3}$ and $y_{4}$. As an error recovery procedure, if the sum of $d_{1}$ and $d_{2}$ turns out to be odd, we know that one of the two roundings of $i_{1}$ and $i_{2}$ has been done in the wrong way: we can take the one which is further away from its closest integer value and re-perform its rounding in the opposite way (decreasing the confidence value accordingly).

As the last step, we plug the $y_{1 \ldots 4}$ into Eq. (3) to get an overdetermined system, which is solved to find the final result $\vec{o}=\left(o_{x}, o_{y}\right)$ for the given pixel: the redundancy is used to reduce errors.

In principle, we could use the confidence values to reject bad or noisy measurements. However, we did not need to in practice, as the matte we computed showed high confidence for all the point with sufficient reflectance $\alpha$, i.e., at all points where the original signal is not severely darkened (see Section 4.3).

\subsubsection{Number of stripes}

The number of stripes used within one pattern is quite important. Ideally, the accuracy of the reconstructed $\vec{o}(u, v)$ will be increased with a higher number of stripes. Assuming that the $x_{i}$ are always determined with the same accuracy no 
matter how many stripes are displayed, they represent a quantity varying at a higher rate resulting in higher precision.

But, if the stripes are too close, the computation of $y_{1 \ldots 4}$ becomes more and more unstable. Since $y_{i}$ are discrete values they are either computed correctly or deviate a lot from the correct solution. Their computation is very robust but if too many stripes are present the system may undergo more errors than it can handle. In these cases it would probably make sense to use the computed $y_{i}$ of neighboring pixels to detect and correct errors, something we do not exploit currently.

In our implementation we use five stripes along the $x$ axis for the vertical pattern and four repetitions for the horizontal one. The diagonal patterns are slanted by $45^{\circ}$, resulting in four repetitions along the $x$ axis. As required four and five are coprimes. Another desired property is that the patterns have more or less the same frequency. At the chosen frequency, the $y_{1 \ldots 4}$ present little or no errors, while the $x_{1 \ldots 4}$ still provides enough precision.

\subsection{Color shift}

Our pattern scheme is naturally resistant against darkening because of the scaling in Step 3 of the algorithm in Section 4.2. That means a good matte can be captured even if captured color values do not sum up to one because only a fraction of the original brightness is reflected by the surface. The ability of the system to work independently of the obtained brightness is important, since it leaves some freedom in factors like camera exposure time, and gives robustness against absorption by the mirroring surfaces.

As another good quality, the zero-order continuity of the used periodic functions (see Fig. 3) gives to the system a relative resistance to the blurring resulting from the aliasing of the CCD.

On the other hand, the use of colored patterns makes the technique prone to errors due to color shift, mainly occurring as a consequence of two factors:

- reflective surfaces that color the light they reflect, such as golden surfaces; or

- differences in the color irradiated by a pixel of the monitor into different directions.

These effects may vary from pixel to pixel over the image.

One way to make the matte acquisition more robust against color shifts is to take a reference image $c_{\alpha}$ of the object reflecting just a white image displayed on the monitor. The color information at each pixel is then used to normalize the subsequent values of the corresponding channels of the pixels $c_{1} \ldots c_{4}$. Color shift due to any of the mentioned reasons will affect both the $c_{\alpha}$ and the other images in the same way. The error can therefore be drastically reduced by a per-pixel, per-channel normalization with $c_{\alpha}$, at the cost of just one extra image. This normalization has proven to be sufficient for our cases. The values of $c_{\alpha}$ are also directly used as the the reflectance channel $\alpha(u, v)$ of the matte. Values that are too dark are clamped to 0 and ignored (they are considered to be either outside the silhouette of the object, or part of the object that is not mirroring, or a part of the object that is not lit by the monitor). 


\section{Geometry reconstruction}

In this section, we describe how the geometry is reconstructed from the acquired environment matte. Like in conventional 3D range scanning (e.g., laser based) the geometry will be recovered as a range map, i.e., at every pixel of the environment matte we compute a depth value (the distance between the principle point of the camera and the surface point visible at the considered pixel).

To reconstruct the geometry of the entire surface of the scanned object, multiple range maps are necessary, each obtained by a single matte captured from a different viewpoint. Since we produce the same output data as in conventional 3D scanning (range maps) the same tools can be used to post-process them to reciprocally align them, merge them, fill holes, and so on $[18,22]$.

The process of deriving a range map from an environment matte, i.e., the core process of shape from distortion, can be seen as a mixture of shape from shading and standard triangulation-based range scanning approaches. The transformation of a normal map into a depth map and vice versa is similar to the problems in shape from shading, where reflectance is used to compute a normal map which further is integrated into a depth map. Our method is also similar to triangulation-based approach, since the depth to normal relation is given by the triangle determined by the camera, the surface point and the active light source, in our case the point of the monitor intersecting the reflected ray.

The major difference, with respect to both shape from shading and triangulationbased range scanning, is that in our case neither depth values nor normal directions are measured directly but rather found by a stable optimization. The depth values are obtained by two major steps. At first, a random initial depth value is assigned to a random point and propagated through the whole matte, at the same time constructing both the depth map and a normal map. Both maps are further refined to obtain a more consistent field. In the second step, we optimize the initial depth value with respect to the self coherence of the surface.

\subsection{Data structures and terminology}

We use the following explicit data structures, each organized as a $2 \mathrm{D}$ map of the same size as the environment matte (which is also the size of the input pictures):

- a distance scalar field $d(u, v)$, coding in each position the distance from the point of view to the surface of the object [along the viewing ray at position $(u, v)$ ];

- a normal field $\vec{n}(u, v)$, coding the normal of that point.

In the rest of this section, we will furthermore use the following terminology:

- as in the previous section, $\alpha(u, v)$ and $\vec{o}(u, v)$ refer to the reflectance and the origin field of the input matte;

- $\vec{r}(u, v)$ denotes the viewing ray through the pixel $(u, v)$ of the matte (obviously this data is not stored, but implicit given by the intrinsic and extrinsic camera parameters) 
- the monitor function $\vec{m}: R^{2} \rightarrow R^{3}$ maps the 2D position of a pixel on the monitor $\vec{o}(u, v)$ to the $3 \mathrm{D}$ position of the pixel in world space. How to determine $\vec{m}$ is described in Section 6.

At the end of the process, the output consists of the distance field $d(u, v)$, optionally enriched by the normal values of $\vec{n}(u, v)$, or/and by the reflectance $\alpha(u, v)$.

\subsection{Converting distance tolfrom normals}

Before solving the entire problem, let us first resolve a pair of related, trivial subtasks.

Let us assume that the current point of view $P \vec{o} V$ is in the origin. If we knew the depth at a single point $d(u, v)$ along the normalized ray $\vec{r}(u, v)$ from the origin as well as the origin of the reflected light on the monitor $\vec{m}(\vec{o}(u, v))$, then surface normal at that position $n(u, v)$ can directly be computed as (see Fig. 4).

$$
\vec{n}(u, v)=\frac{1}{2}\left(\frac{(\vec{m}(\vec{o}(u, v))-d(u, v) \cdot \vec{r}(u, v))}{\|(\vec{m}(\vec{o}(u, v))-d(u, v) \cdot \vec{r}(u, v))\|}-\vec{r}(u, v)\right) .
$$

Likewise, given $\vec{n}(u, v)$ we can compute the only value for $d(u, v)$ that satisfies

$$
d(u, v) \cdot \vec{r}(u, v)=(\vec{m}(\vec{o}(u, v))-k(2 \vec{r}(u, v) \cdot \vec{n}(u, v)) n(\overrightarrow{u, v})+\vec{r}(u, v))
$$

for some $k<0$. In other words, the knowledge of the value of the matte $\vec{o}(u, v)$ allows us to uniquely compute the normal from the depth of the surface point visible at $(u, v)$, and the other way around (see Fig. 5).

There also exists a second way to deduce either of the two fields $\vec{n}(u, v)$ and $d(u, v)$ from the other under certain assumptions about surface continuity (see Section 5.4.1): given the values of $\vec{n}(u, v)$ we can integrate them to obtain the depth field $d(u, v)$ propagating an initial depth estimation, similarly to what is done in shape from shading approaches $[3,25]$. Likewise, given the depth values $d(u, v)$ one can eas-

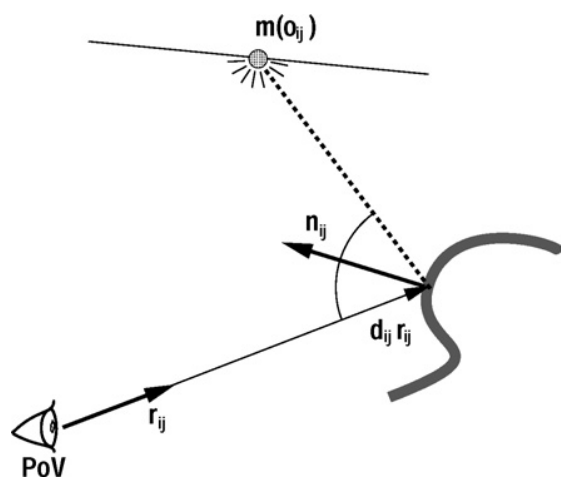

Fig. 4. Relationship between the surface normal $\vec{n}(u, v)$ and distance $d(u, v)$ (along the ray $\vec{r}(u, v)$ given the 3D position $\vec{m}(\vec{o}(u, v))$ of the light origin (the monitor pixel at position $\vec{o}(u, v)$ ). 


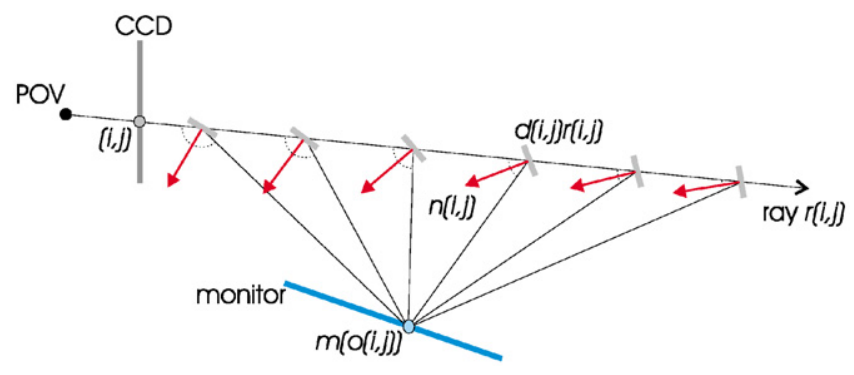

Fig. 5. Given a ray and a 3D position of the light source, a one-dimensional space of solutions, consisting of a surface normal and a distance, are possible.

ily derive the normal at one point, e.g., as the normal of a plane fitted to the depth values of neighboring pixels.

\subsection{Depth extraction}

Unfortunately, we know initially neither the values of $d(u, v)$ nor $\vec{n}(u, v)$. An initial approximation of the two fields is found by propagating an initial guess for the depth value at one point through the whole matte:

(1) Start with an arbitrarily chosen initial point $\left(u_{0}, v_{0}\right)$ inside the matte and assign a random distance $d\left(u_{0}, v_{0}\right)$ to it. How to determine a proper initial depth value is explained in Section 5.4. A boundary $\Gamma$ is initialized consisting of only the point $\left(u_{0}, v_{0}\right)$.

(2) Compute the normals using point-wise Eq. (7) of all boundary points.

(3) Compute the depth $d\left(u_{n}, v_{n}\right)$ of every untouched point $\left(u_{n}, v_{n}\right)$ neighboring to any boundary point $\left(u_{\Gamma}, v_{\Gamma}\right)$, based on the depth value $d\left(u_{\Gamma}, v_{\Gamma}\right)$ using the surface tangent implicitly given by $\vec{n}\left(u_{\Gamma}, v_{\Gamma}\right)$. Add the point $\left(u_{n}, v_{n}\right)$ to the boundary. If a point does not have any untouched adjacent points, remove it from the boundary.

(4) Repeat from 2 until all points are processed.

For a more robust algorithm, before processing a point in Step 3, we wait until $k \geqslant N_{\mathrm{p}}$ neighboring points around a given point $d\left(u_{n}, v_{n}\right)$ have been processed (apart from the initial one). Its depth value is then computed as the average of the depth predicted as above, estimated by the $k$ different directions. This produces more accurate results and is also a natural way to filter out error prone points: if a given point is never reached at least $N_{\mathrm{p}}$ times, we just drop it from the scan. In our implementation we used $N_{\mathrm{p}}=3$ out of the eight neighbors surrounding one point.

In a second phase, we refine the initial approximation of both fields exploiting the dependency of one point on its complete neighborhood until two consistent fields are found: 
(1) integrate the current normal field $\vec{n}(u, v)$ into the distance field $d(u, v)$;

(2) $\forall(u, v)$ use Eq. (7) to compute the new $\vec{n}(u, v)$ from the current $d(u, v)$;

(3) repeat from 1 until convergence.

In this second phase, we take into account the surface continuity along all directions, as opposed to the $N_{\mathrm{p}}$ directions during the boundary propagation in the initial phase.

Note that both phases are based in a point-wise computation of $\vec{n}$ from $d$ [using Eq. (7)], and a local conversion of $n$ to $d$ (using normal integration). In theory, we could have used a dual approach that uses Eq. (8) instead to obtain $d$ from $\vec{n}$ per pixel, in conjunction with a derivation process (fitting normals to the depth field) to locally determine $\vec{n}$ from $d$. We prefer the former approach since both the involved computations seem to be more robust than the ones of the latter.

\subsection{Initial depth}

The discussed algorithm proved to converge fast in all tested cases. Unfortunately it converges "too much": starting with different initial guesses of the depth $d\left(u_{0}, v_{0}\right)$ at an arbitrary chosen initial point, different surfaces are reconstructed. In fact, the more the initial point is assumed to lie far from the camera, the more its normal is reconstructed as constantly pointing towards the camera: it is easy to see that $\vec{n}(u, v)$ in Eq. (7) converges to $-\vec{r}(u, v)$ when $d(u, v)$ tends to infinity. Therefore, if the initial depth is overestimated, neighbor points are reconstructed as lying on a plane orthogonal to the camera, propagating the overestimation over all the surface; conversely if the initial depth is underestimated, the reconstructed normal will point away from the camera, leading to a too strongly curved reconstruction of the surface. In both cases, the method will converge, but to a wrong surface reconstruction.

In other words, the per-point ambiguity shown in Fig. 4 results globally in an ambiguity between large, more or less flat objects far away or small, strongly curved objects close to the camera (see Fig. 6).

The ambiguity consists of a single undetermined scalar value for the entire patch. We aim at solving it without requiring anything more than the information present in a single matte. Although the correct depth could easily be recovered by a second matte done with a second monitor position, this would increase the acquisition time and effort considerably since the monitor position has to be changed and thus recalibrated at each scan (it is not possible to just use two monitors, switching them on one at a time, because, in order for them to light two non-disjoint mattes, they would require a positioning that would make one obscure the other).

The basic fact we will exploit to obtain the correct depth with just a single matte is that not all of the possible shapes solving the ambiguity are equally self coherent. The form of incoherence we address is the one depicted in Fig. 7, where the depth estimate of a given points $(A)$ along a ray $\vec{r}_{0}$ is propagated along two different paths, namely $A B C$ and $A B^{\prime} C^{\prime}$, to the same ray $\vec{r}_{2}$, but leading to different depth values at that ray. For each of the two paths the depth along the starting ray determines the normal at that point, which in turn determines the delta of depth to the next ray. As 


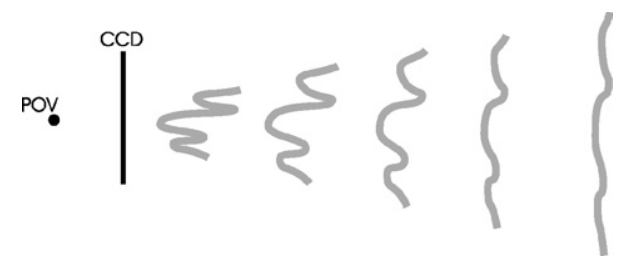

Fig. 6. Intuitive sketch showing the relationship of the overall shape, size, and position of the resulting range scan depending on the initial depth estimate.

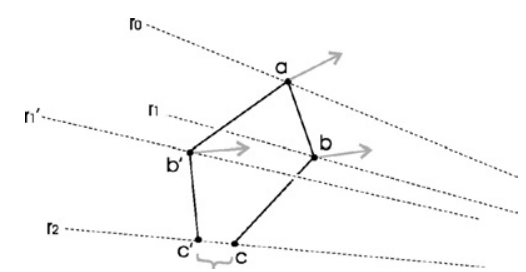

Fig. 7. The basic case of incoherence that must be summed over the whole surface in all directions to evaluate the total incoherence.

already described, both in the initial estimation and in the refinement phases we average the $C$ and $C^{\prime}$ depth values of any considered propagation directions to compute the final, most consistent depth value for $C$. However, the variance of the values for different paths can be used as the measure of incoherence we need. This measure is further integrated over all points in the matte.

Plotting a graph of this error measure versus the initial depth estimate (e.g., Fig. $8 \mathrm{~A})$ we always observed a well defined minimum. This minimum determines the correct depth. Since there is no obvious way to treat the function in an analytical way, we need to find this minimum with an iterative method.
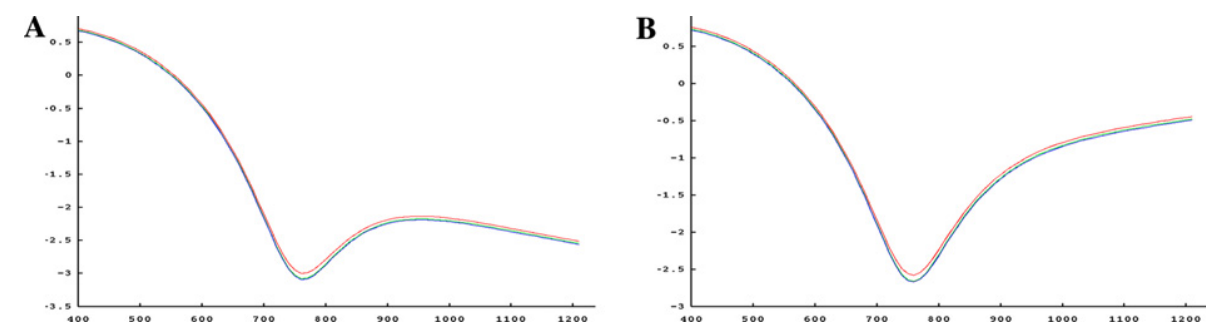

Fig. 8. A plot of the computed incoherence integrated over the surface and all directions ( $y$ axis, in logarithmic scale) versus the initial depth estimation ( $x$ axis, in $\mathrm{mm}$ ). A minimum clearly exists, corresponding to the real distance of the object from the camera. The three plotted lines correspond to the incoherence of the surface right after the initial estimate, after one refinement, or after five refinements (note that the error decreases but the abscissa of the minimum stays the same). The function is shown without (A) and with (B) an extra weighting to take into account the flattening effect for far away objects (see text). 
A problem is that the error function tends to zero when the initial distance parameter goes to infinity. In fact, if the object is far enough then all the normals will approximately point towards the viewer (see Fig. 6) and the normal map will be artificially coherent. A natural solution is to weight the measured incoherence with the inverse of the dot product of the view point and the normal computed at the initial point with that depth, that is, the inverse of the typical delta of depth between two neighboring points. With this adjustment the error function proved to have just a single local minimum (see Fig. 8B), so we can apply the Golden Section Search method to find the minimum with great accuracy using few iterations. The weighting sometimes moves the computed minimum by a fraction of a millimeter, but we believe that this estimate is more accurate.

Time can be saved if during each iteration of the Golden Ratio Search only the initial step of the matte to depth map conversion (see Section 5) is executed. The refinement iterations afterwards reduce the error but we never observe any significant change of the position of the minimum (see Fig. 8).

This measure of self coherence makes intuitively sense and gives good results, that is, the estimated distance to the camera is comparable, but much more accurate, to those that we measured with any empirical method on the physical object. We tested the overall accuracy of the method by using our scanning technique over a known object (see Section 7.2).

Although the method produces highly accurate results it unfortunately breaks down for too small regions. If one region consists of too few connected, usable points the measure of self coherence is no longer reliable, reducing the overall accuracy.

\subsubsection{Continuity assumption}

During both the normal-to-depth or depth-to-normal conversion, the algorithm assumes that the surface is first-order continuous.

In practice, we do not really have to worry about gradient discontinuity (angles that are totally steep): real object scans never present them and even when the object itself present very steep angles, these are made a little smoother by the blurring inherent in the image acquisition system. In fact, the problem only occurred when testing the technique on synthetic images, never when using real pictures.

Another case of discontinuity arise for the zero-order (apparent) depth discontinuity due to self-occlusion. Typically depth discontinuities can be observed as separated regions/patches in the matte. At a depth discontinuity the normal of the surface will almost invariably be oriented orthogonally to the view direction, and therefore during matte acquisition it would not show the reflection of the monitor, which in all useful configuration is in front, not behind, the object with respect to the camera. Parts of the object not reflecting the monitor are black in the matte and are therefore automatically ignored by the algorithm.

\subsubsection{Disjoint patches}

If the extracted matte presents more than one connected region of usable pixels (we will refer to them as matte patches), than each of them will be handled separately, each with a differently determined optimal initial depth. The result will be dif- 
ferent patches within the same range and normal map. These patches, even if they are disjointed, are reciprocally aligned (that is, their relative position is fixed), and they can be treated as a single scan for any further processing if they are large enough for a reliable depth estimation.

\subsubsection{Interreflections}

One more issue is how to solve the problem caused by multiple reflections. The matte captures the reflections of a reflection as well as a simple reflection. In principle, if properly exploited, this could be an advantage rather than a problem, since double reflections provide information on both surfaces reflecting the ray. However, observations are clearly troubled by ambiguity and it is not clear how to analyze them. It would be important, if not to exploit multiple reflections, to at least detect and ignore them.

Some factors make detection possible. First of all, parts of the object lighted (in the pictures, and therefore in the matte) by multiple reflections tend to be separated from those showing single reflections. So, in many cases, either the captured colors of all pixels in the patch are due to multiple reflections or all pixel values are due to a single reflection. Another observation is that a patch caused by multiple reflections produces depth maps that show an incoherence (in the sense explained in Section 8) several order of magnitude bigger than single reflection patches. Finally, multiple reflection patches can also be significantly darker, in cases where the object absorbs part of the visible light it reflects. At present, we did not experiment using some, or a combination, of these factors to automatically detect patches plagued by multiple reflection.

\section{Calibration}

To correctly interpret the measured data, it is necessary to perform the following calibration steps:

- calibration of the intrinsic camera parameters,

- calibration of the response curve of the monitor/camera combination,

- calibration of the monitor position and shape relative to the camera.

The first two steps must be done only once per choice of equipment (camera, monitor) and per lens setting whereas the position of the monitor must be determined at the beginning and whenever one of the two is moved. While our method has been designed so that a given position of camera-monitor could be easily used for multiple scans by just moving the target object, sometimes it may be desirable to move one of them to get a larger scan (i.e., more of the target object reflecting the monitor).

\subsection{Intrinsic camera parameters}

The intrinsic camera parameters such as focal length, lens distortion, and central projection point must be known to relate a pixel position in the image to a position 
on space. It is not sufficient to rely on data in the camera and lens specification as the focal length changes, for example, with the subject distance. We used a standard method similar to Zhang et al. [26] to recover the intrinsic parameter of our camera system.

\subsection{Photometric calibration}

Our approach uses color information to infer the screen position that illuminates a surface point. Similar to a shape from shading system, we therefore have to ensure that the acquisition system is photometrically calibrated. In particular, we have to ensure that the system is linear, i.e., that an image of a linear ramp displayed on the monitor is captured by the camera system again as a linear ramp. Furthermore, crosstalk between the color channels in the captured images should be avoided. For our scanner, we therefore have to calibrate both the display and the acquisition system. Within our imaging pipeline there are two places that require calibration:

- the display system where the RGB values of pixels in the original patterns are interpreted by the computer (which might include application of a gamma factor or an ICC profile) and displayed on the monitor

- the acquisition system where the camera will normally detect a different RGB signal as the spectral responses of the CCD sensors do usually not match the spectral emission of the monitor phosphors. After some color processing by the camera (possibly including the application of a response curve), the raw image signal is available.

To calibrate the display system, we first determine the response curve resp. the opto-electronic conversion function (OECF) of the camera using some standard method (e.g., [27-29]). If the camera does not have a linear response, applying the inverse of the response curve transfers the images into a linear camera color space.

To remove crosstalk between the three color channels, which is mainly caused by the different spectral characteristics of the monitor and the camera, a simple basis transform in the linear camera color space is sufficient. An image such as Fig. 9 containing pure red, green, and blue color patches is displayed on the monitor, captured with the camera and linearized. The corresponding image values $R, G$, and $B$ are assembled in a basis transform matrix $M$. The inverse of $M$ can be used to separate the colors:

$$
\left(\begin{array}{c}
r_{\text {sep }} \\
g_{\text {sep }} \\
b_{\text {sep }}
\end{array}\right)=\left(\begin{array}{c}
r_{\text {cam }} \\
g_{\text {cam }} \\
b_{\text {cam }}
\end{array}\right)^{\mathrm{T}} M^{-1}=\left(\begin{array}{c}
r_{\text {cam }} \\
g_{\text {cam }} \\
b_{\text {cam }}
\end{array}\right)^{\mathrm{T}}\left(\begin{array}{ccc}
R_{\mathrm{r}} & R_{\mathrm{g}} & R_{\mathrm{b}} \\
G_{\mathrm{r}} & G_{\mathrm{g}} & G_{\mathrm{b}} \\
B_{\mathrm{r}} & B_{\mathrm{g}} & B_{\mathrm{b}}
\end{array}\right)^{-1} .
$$

In theory, this calibration depends also on the reflective material of the target object, different materials requiring different separation. In practice, we found out that, using the precautions about color shift presented in Section 4.3, a single matrix is usually good enough for any material.

To calibrate the display system, we have to capture the response curve of the monitor by displaying a linear ramp for each color channel varying from 0 to the 


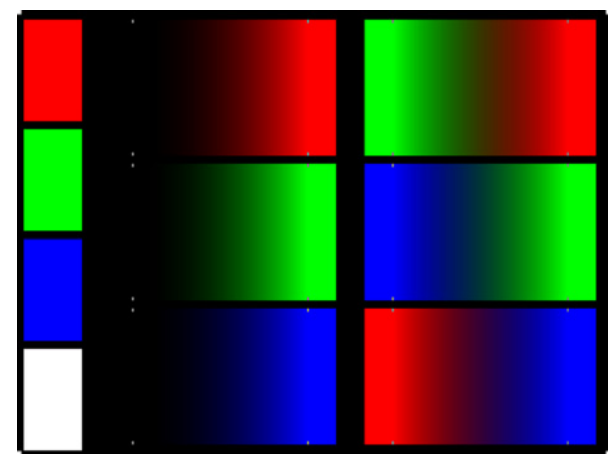

Fig. 9. Test image used for the calibration procedure. Left: pure red, green, blue, and white color patches. Middle: single color ramps. Right: dual color ramps as used in the patterns. (For interpretation of the references to color in this figure legend, the reader is referred to the web version of this paper.)

maximal value (see Fig. 9, middle). By inverting the captured ramp, we can now generate patterns that are displayed as linear ramps on the monitor and are captured and interpreted as linear ramps with a clear separation of the channels.

Any error made (or not compensated for) by the above calibration steps can lead to artifacts such as crosstalk between the color channels and to non-linearities in the captured matte. The quality of the calibration can therefore be judged by analyzing images of dual color ramps which are used in the patterns (see Fig. 9, right). During our experiments, we achieved a high linearity of the linear ramps and a good separation of the color channels as can be seen in Fig. 10.

\subsection{Geometric calibration}

In the reconstruction phase, we need to know the monitor function $\vec{m}$ (see Section 5), that is, the exact location of each pixel on the monitor relative to the camera to interpret the captured mattes correctly. This task can be split in two parts: modeling the position of each pixel relative to the monitor (a one-time calibration) and recov-
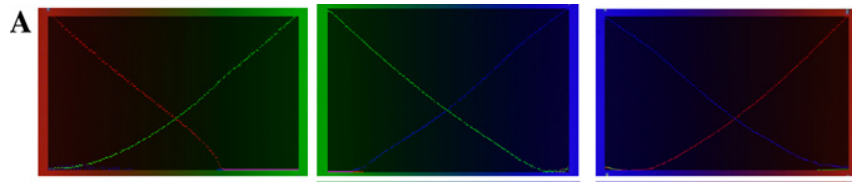

B
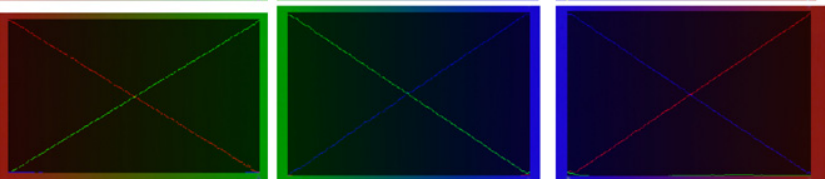

Fig. 10. The three color ramps red to green, green to blue, and blue to red. (A) Their first approximation, i.e., linear ramps sent to the monitor and captured by the camera lead to irregular, nonlinear functions. (B) The final ramps after calibration are linear and well separated. (For interpretation of the references to color in this figure legend, the reader is referred to the web version of this paper.) 
ering the position of the monitor. In our case, it was sufficient to model the monitor as a flat rectangle, with pixels evenly spaced on it. If the monitor is not flat or regular enough, it might be necessary to model the shape of the monitor more accurately.

An obstacle in detecting the monitor position is the fact that typically the monitor is not directly visible from the camera. Instead, both the monitor and the camera are facing a similar direction, so that the camera can capture monitor light reflected by the target object. The monitor position measurement can still be easily performed using a single picture, with the aid of some mirroring sample objects with known, very simple, geometry such as mirroring spheres.

Given their perceived size and position in a captured image (and the knowledge of their real world size, plus the intrinsic camera parameters), it is easy to compute 3D position of the spheres, relatively to the camera, using basic projective geometry (see, for example [30]). Then, the image position of the reflection over the sphere of each corner of the monitor is detected. For each such position we compute the 3D reflected ray that starts from the corresponding point of the sphere, and that must pass through the monitor corner. Using several spheres in a single image, it is possible to compute the $3 \mathrm{D}$ positions of the monitor corners as the intersection of these rays. The monitor (modeled as a rectangle of known dimensions) is fitted in those corners. A similar method could be designed using a flat rectangular mirrors of known size instead of the spheres.

For our experiments, we used two metal spheres with $60 \mathrm{~mm}$ diameter for the calibration. The spheres are roughly located at the same position and have approximately the same size as the objects to be scanned. This way it is possible to capture the matte after the position calibration without changing the focus setting of the lens, which would invalidate the intrinsic calibration.

The calibration remains valid as long as the camera and the monitor are left in place. Fortunately, our technique requires a single matte (which is captured with images all shot leaving both the monitor and the camera in the same position), to extract a range scan. Given that the object can be moved, it is possible to keep the camera and monitor fixed and just rotate the object to perform all the needed scans.

\section{Results}

In this section, we present some results obtained using our technique to scan three objects - a polished metal sphere with $60 \mathrm{~mm}$ diameter and two identical plumbery objects, to one of which we added some artificial dents. As the two phases of our algorithm can also be used separately, we will present the results for each phase individually.

\subsection{Matte capture and extraction}

Our measurement setup consists of a standard 19 in. CRT monitor for displaying the patterns and a high quality Kodak DCS 560 digital still camera (see Fig. 11). Due to their huge view dependent color shift, most current flatscreen monitors cannot be used for display although they would be much lighter and easier to move during set- 


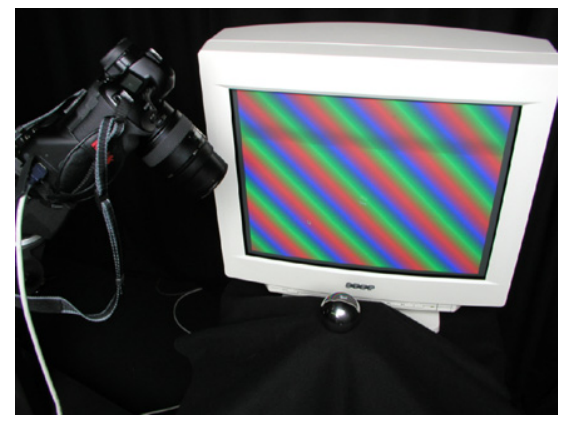

Fig. 11. The setup used during our experiments. The patterns are displayed on a $19 \mathrm{in.} \mathrm{CRT} \mathrm{monitor} \mathrm{and}$ reflected off the target object (here the metal sphere in front of the monitor). The images are captured with a high quality digital still camera.

up. All experiments have been performed in our photo studio [31] which has been specially designed to keep the influence of the environment on such measurements as low as possible.

Fig. 12 shows some results of our matte capture and extraction algorithm. At first, we show the object reflecting the white monitor image $c_{\alpha}(\mathrm{A})$, and two of the four patterns $c_{1}, c_{4}(\mathrm{~B}, \mathrm{C})$. To illustrate the quality of the extracted matte, we colored each of its points using the reconstructed origin field $\vec{o}(u, v)$ to index a checker pattern (D). Note that the stripes on the sphere are of equal width proving that there is no systematic error in our system when deriving the position of the reflected monitor pixel from image colors. The irregularity in the the middle of the image corresponds to a real, extremely tiny imperfection of the used mirroring sphere. In subfigure (E), a photograph instead of the checker pattern is used for the mapping. The resulting image can be compared with a real picture showing the original object reflecting the same photograph displayed by the monitor (F). Apart from a color mismatch which we did not correct for, the two images are almost identically.

All mattes have been computed on a standard PC (Athlon $1.4 \mathrm{GHz} 512 \mathrm{MB}$ ). Computing a matte with over $100 \mathrm{~K}$ points takes less than a second.

\subsection{Geometry reconstruction}

A summary of the results of the geometry reconstruction phase is given in Fig. 13. To assess the accuracy of the reconstructed geometry, we needed to use, as a test target, an object with a shape that was exactly known a priori: we resorted to a $60 \mathrm{~mm}$ metal sphere (the sphere are used for other calibration purposes and the estimation of their diameter is known to be very accurate). As the table shows, in a range-scan of $75 \mathrm{~K}$ range points the RMS to an optimal sphere with $60 \mathrm{~mm}$ diameter is as small as $21 \mu \mathrm{m}$. Fig. 14 shows how the error is distributed over the scan of the sphere. The scan shows a very high accuracy and very low noise. We believe that these small errors are mainly due to inaccurate intrinsic camera parameters, and secondarily due to the errors in the monitor position estimation. 

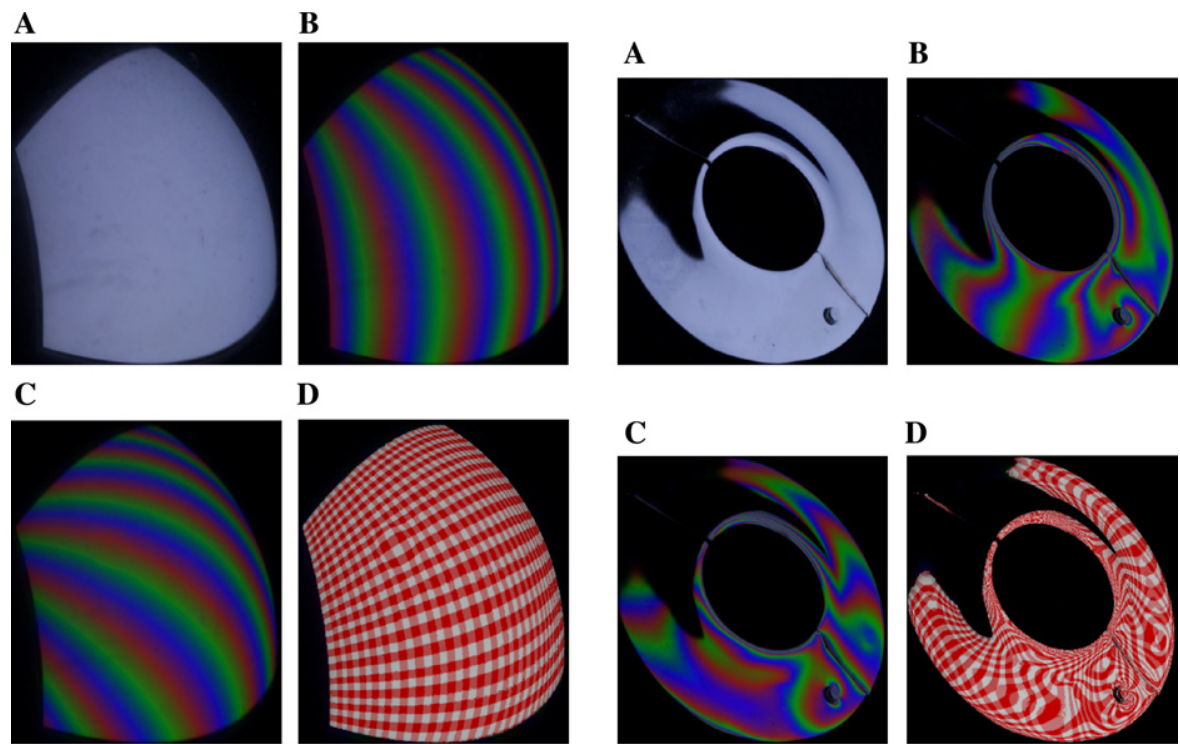

D
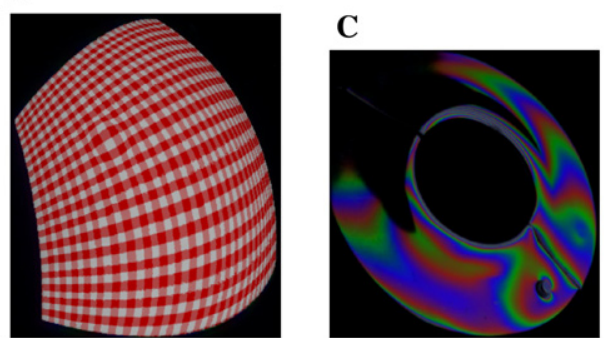

D

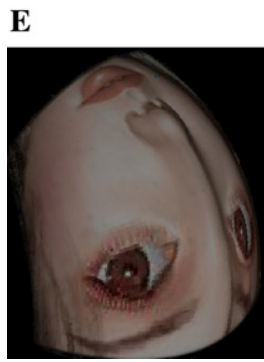

$\mathbf{F}$

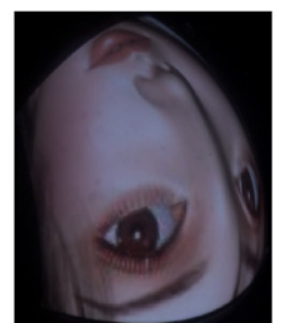

$\mathbf{E}$
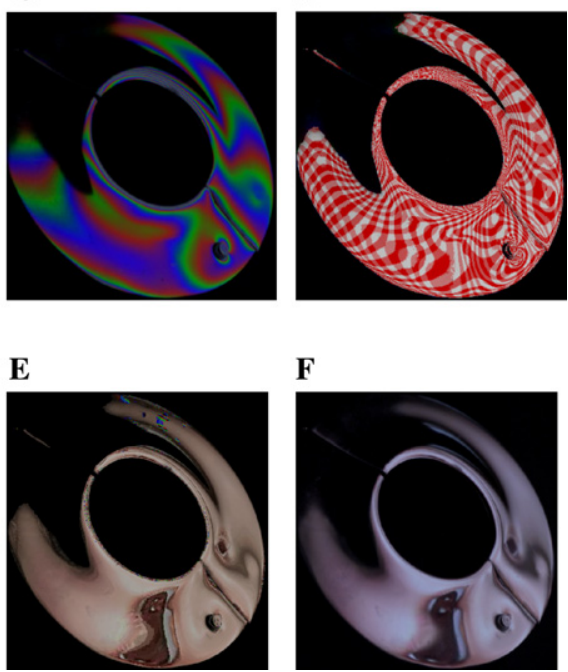

$\mathbf{F}$

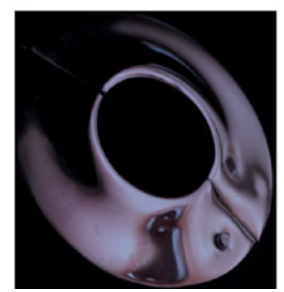

Fig. 12. Matte captured for a mirroring sphere and a plumbery object. Subfigures (A), (B), and (C) show the input images reflecting patterns $c_{\alpha}, c_{1}, c_{4}$. In subfigure (D) and (E), the captured matte is shown using the origin field $\vec{o}(u, v)$ to index a checkered board (D), or an image (E). As reference, $(\mathrm{F})$ shows pictures of the real objects mirroring the image. See Fig. 16 for another example.

As a test for a more complicated object consisting of multiple, independent patches, we reconstructed the geometry of the plumbery objects. For the first one without dents whose matte is visible in Fig. 12, we show the reconstruction of the normal field $\vec{n}(u, v)$ and the distance field $d(u, v)$ in Fig. 15. The figure further displays the corresponding geometry (rendered adding a trivial connectivity to the range points identified by the distance field). Finally, in Fig. 16 we show some results for the dented plumbery object, concerning both the acquired matte and the reconstructed geometry.

\section{Conclusion}

The presented method is a large step towards recovering the surface geometry of a mirroring surface - an important problem not only in computer graphics but also in 


\begin{tabular}{|l|c|c|c|}
\hline & $\begin{array}{c}60 \mathrm{~mm} . \\
\text { sphere } \\
\text { matte }\end{array}$ & $\begin{array}{c}\text { plumbery } \\
\text { object } \\
\text { (clean) }\end{array}$ & $\begin{array}{c}\text { plumbery } \\
\text { object } \\
\text { (dented) }\end{array}$ \\
\hline \hline Number of pixels & $75 \mathrm{~K}$ & $38 \mathrm{~K}$ & $108 \mathrm{~K}$ \\
\hline Number of Patches & 1 & 2 & 2 \\
\hline \hline Time Initial Depth Est. $(s)$ & 11.9 & 7.6 & 20.0 \\
\hline Time Refinement $(s)$ & 5.2 & 3.0 & 7.5 \\
\hline Time Total $(s)$ & 17.1 & 15.1 & 27.5 \\
\hline \hline Est. Initial Depth $(\mathrm{mm})$ & 756.716 & 819.640 & 998.426 \\
\hline Measured Coherence before Refinement $(\mathrm{nm})$ & 385 & 284 & 220 \\
\hline Measured Coherence after Refinement $(\mathrm{nm})$ & 275 & 263 & 210 \\
\hline RMS error $(\mu m)$ & 21 & - & - \\
\hline
\end{tabular}

Fig. 13. Results for three test cases: a matte of the metal sphere, of the plumbery object, and of the dented plumbery object. When multiple patches are present, all data refer to the computation of the largest patch. The coherence row reports, in nanometers, the quantity described in Section 5.4 before it is weighted, averaged over all valid scan points. The number of pixels corresponds also to the number of range points in the resulting range map. The errors for the plumbery objects cannot be quantified for lack of reference data.

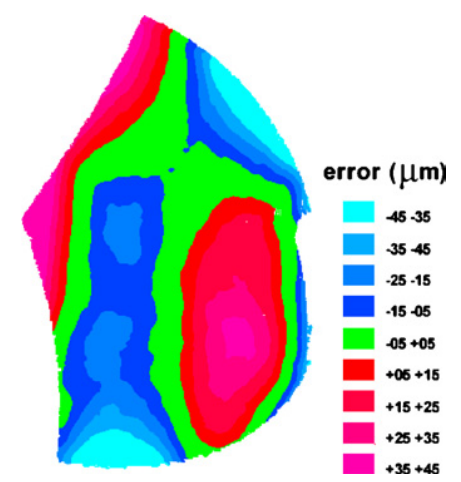

Fig. 14. Error distribution in a scan of a sphere with $60 \mathrm{~mm}$ diameter similar to Figure 12 left. The error is shown as signed distance to an optimal sphere in micrometer.

industrial applications [1]. Due to the basic acquisition principle (an environment matte is related to the first derivative of a surface), the achieved precision is very high and even small details can be recovered accurately.

The acquired scans are range maps that can be dealt directly with existing, well studied techniques developed in other contexts of range scanning. The acquisition time for a single scan is quite reasonable and only standard, cheap hardware components are required. 

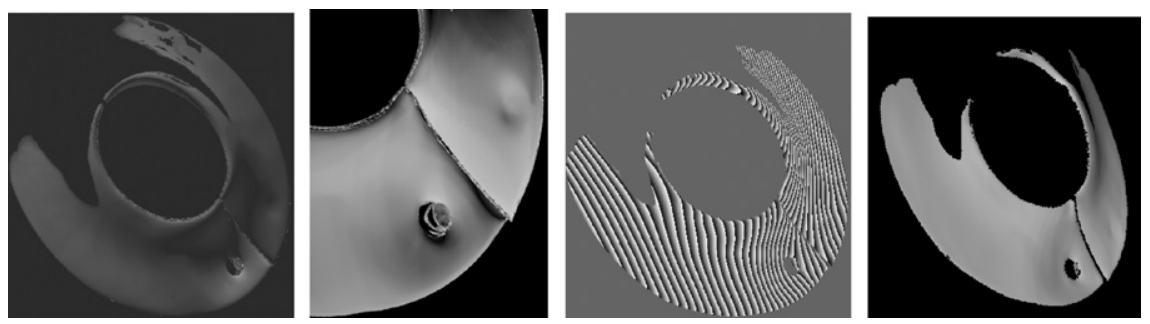

Fig. 15. Geometry reconstructed from the extracted matte of the plumbery object visible in Fig. 12. From left to right: the close-up of the normal field $\vec{n}(u, v)$ rendered with Lambertian surface properties reveals even small details. The distance field $d(u, v)$, visualized with isovalued lines. The resulting geometry, $32 \mathrm{~K}$ faces, rendered from a slightly different point of view. (see Fig. 16 for another example.)
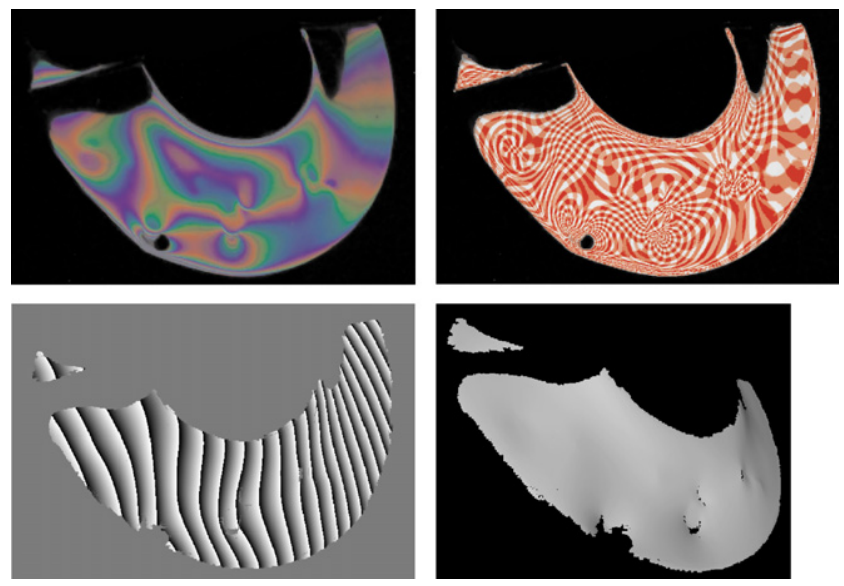

Fig. 16. Results of measuring the dented plumbery object. From top left to bottom right: one of the original pictures shot to capture the matte, the extracted matte indexing a checkered pattern, the resulting depth map (isolines) and a rendering of the reconstructed geometry.

The technique consists from two components that can also be used independently and in different contexts: the environment matting scheme and the reconstruction of the surface from the environment matte. The proposed environment matting scheme shows an efficient way to improve the precision of environment matting to sub-pixel accuracy. In cases where the matte extraction is difficult due to the reflection properties of a targeted object, using a more slow and robust environment matting method can improve the acquired matte. Such a matte can nevertheless be used as input for the surface reconstruction.

\section{Future work}

The size of the scanned area is limited by the fact that only surface points are scanned for which the reflection of the monitor is seen by the camera (a limitation 
similar to the ones common in triangulation based 3D scanners). An easy extension of our work would therefore be to replace the monitor with a hemispherical projection screen or a cave. In principle, this requires only an additional calibration phase to recover the geometry of the projection screens. However, this would increase dramatically the cost of the required hardware.

This limitation poses also new problems on the still largely open field of scan planning: to maximize the surface area acquired by a single scan, one must maximize the portion of the object reflecting the monitor into the camera. A locally optimal position could be automatically found by some appropriate search. Such an automated planning system be especially useful in combination with a robot controlled acquisition environment [32].

One more issue is how to deal with multiple reflections. As already noted, this, if properly exploited, could be an advantage rather than a problem, since double reflections provide information on both surfaces reflecting the ray. But, while detecting multiple reflections is comparatively easy (as discussed in Section 5.4.3), it is quite difficult to integrate them into a coherent depth map.

Another promising research issue consists in the possible use of a similar shape from distortion approach to reconstruct transparent objects such as glass or some types of plastics. The basic concept would be to capture a matte with light from the monitor shining through the object. Its shape is reconstructed by looking at the distortions caused by refraction and reflection. This would be a further significant and very useful extension of the scope of 3D acquisition in general, as transparent objects represent, similarly to mirroring ones, an extremely troublesome yet needed category. It will likely be very challenging to reconstruct the geometry, partly because refractions are more complicated then reflections, partly because each light ray affected by the object at all is likely to undergo multiple refractions and reflections during its monitor-to-camera path. As a side effect, work in this direction could be helpful in exploiting and using multiple reflection matte patches.

\section{References}

[1] F. Chen, G.M. Brown, M. Song, Overview of three-dimensional shape measurement using optical methods, Opt. Eng. 39 (1) (2000) 10-22.

[2] M. Baba, K. Ohtani, M. Imai, T. Konishi, New laser rangefinder for three-dimensional shape measurement of specular objects, Opt. Eng. 40 (1) (2001) 53-60.

[3] R. Zhang, P.-S. Tsai, J.E. Cryer, M. Shah, Shape from shading: a survey, IEEE Trans. Pattern Anal. Mach. Intell 21 (8) (1999) 690-706.

[4] C.R. Dyer, Foundations of Image Understanding, Kluwer, London, 2001, Chapter 16, pp. 469-489.

[5] M. Oren, S.K. Nayar, A theory of specular surface geometry, Int. J. Comput. Vision 24 (2) (1996) $105-124$.

[6] J.Y. Zheng, Y. Fukagawa, N. Abe, 3D surface estimation and model construction from specular motion in image sequences, IEEE Trans. Pattern Anal. Mach. Intell. 19 (5) (1997) 513-520.

[7] J.Y. Zheng, A. Murata, Acquiring a complete 3D model from specular motion under the illumination of circular-shaped light sources, IEEE Trans. Pattern Anal. Mach. Intell. 22 (8) (2000) 913-920.

[8] P.N. Belhumeur, D.J. Kriegman, Shedding light on image-based rendering, Tech. Rep. CSS-9903, Yale University, Yale, NH, USA (November 1999). 
[9] K. Robinson, Laser measures shiny objects in 3-D, Photon. Spectra 35 (6) (2001) 28-29.

[10] H. Hagen, S. Hahmann, T. Schreiber, Y. Nakayiama, B. Wördenweber, P. Hollemann-Grundstedt, Surface interrogation algorithms, IEEE Comput. Graph. Appl. 12 (5) (1992) 53-60.

[11] M.A. Halstead, B.A. Barsky, S.A. Klein, R.B. Mandell, Reconstructing curved surfaces from specular reflection patterns using spline surface fitting of normals, in: Proceedings of ACM Computer and Graphics Interactive Techniques (SIGGRAPH'96), New Orleans, 1996, pp. 335-342.

[12] W.S. Park, H.S. Cho, Measurement of the 3-dimensional shapes of specular objects using recursive triangulation, in: Proceedings of Pacific Conference on Manufacturing (PCM'96), Seoul, Korea, vol. 1, 1996, pp. 389-394.

[13] S. Savarese, P. Perona, Local analysis for 3D reconstruction of specular surfaces, in: Proceedings of IEEE International Conference on Computer Vision (ICCV’01), Vancouver, Canada, 2001, pp. 594 603.

[14] S. Savarese, P. Perona, Local analysis for 3D reconstruction of specular aurfaces-Part II, in: Proceedings of European Conference on Computer Vision (ECCV'02), Copenhagen, Denmark, 2002.

[15] T. Bonfort, P. Sturm, Voxel carving for specular surfaces, in: Proceedings of 9th International Conference on Computer Vision (ICCV'03), 2003, pp. 591-596.

[16] D.E. Zongker, D.M. Werner, B. Curless, D.H. Salesin., Environment matting and compositing, in: Proceedings of ACM Computer Graphics and Interactive Techniques (SIGGRAPH'99), Los Angeles, 1999, pp. 205-214.

[17] Y. Chuang, D.E. Zongker, J. Hindorff, B. Curless, D.H. Salesin, R. Szeliski, Environment matting extensions: towards higher accuracy and real-time capture, in: Proceedings of ACM Computer Graphics and Interactive Techniques (SIGGRAPH'00), New Orleans, 2000, pp. 121-130.

[18] K. Pulli, Multiview registration for large data sets, in: Proceedings of International Conference on 3D Digital Imaging and Modeling (3DIM'99), Ottawa, Canada, 1999, pp. 160-168.

[19] S. Rusinkiewicz, M. Levoy, Efficient variants of the ICP algorithm, in: Proceedings of International Conference on 3D Digital Imaging and Modeling (3DIM'01), Quebec City, Canada, 2001, pp. 145-152.

[20] B. Curless, M. Levoy, A volumetric method for building complex models from range images, in: Proceedings of ACM Computer Graphics and Interactive Techniques (SIGGRAPH'96), Los Angeles, 1996, pp. 303-312.

[21] C. Rocchini, P. Cignoni, F. Ganovelli, C. Montani, P. Pingi, R. Scopigno, Marching intersections: an efficient resampling algorithm for surface management, in: International Conference on Shape Modeling and Applications (SMI'2001). Genova, Italy, 2001, pp. 296-305.

[22] P. Cignoni, C. Montani, R. Scopigno, A comparison of mesh simplification algorithms., Comput. Graph. 22 (1) (1998) 37-54.

[23] A.R. Smith, J.F. Blinn, Blue screen matting, in: Proceedings of ACM Computer Graphics and Interactive Techniques (SIGGRAPH'96), New Orleans, 1996, pp. 259-268.

[24] C. Rocchini, P. Cignoni, C. Montani, P. Pingi, R. Scopigno, A low cost optical 3D scanner based on structured light, in: Proceedings of Annual Conference of the European Association for Computer Graphics (EUROGRAPHICS 2001), Manchester, UK, 1999, pp. 299-308.

[25] R. Klette, K. Schlüns, A. Koschan, Computer vision: three-dimensional data from images, Springer, 1998.

[26] Z. Zhang, Flexible camera calibration by viewing a plane from unknown orientations, in: International Conference on Computer Vision (ICCV'99), Corfu, Greece, 1999, pp. 666-673.

[27] M.A. Robertson, S. Borman, R.L. Stevenson, Dynamic range improvement through multiple exposures, in: Proceedings of the International Conference on Image Processing (ICIP'99), IEEE, 1999, pp. 159-163.

[28] P. Debevec, J. Malik, Recovering high dynamic range radiance maps from photographs, in: Proceedings of ACM Computer Graphics and Interactive Techniques (SIGGRAPH'97), Los Angeles, 1997, pp. 369-378.

[29] ISO 14524 Photography-electronic still picture cameras methods for measuring opto-electronic conversion functions (OECFs) (1999).

[30] A. Criminisi, I. Reid, A. Zisserman, Single view metrology, in: Proceedings of 7th International Conference on Computer Vision (ICCV’99), Kerkyra, Greece, 1999, pp. 434-442. 
[31] M. Goesele, W. Heidrich, H. Lensch, H.-P. Seidel, Building a photo studio for measurement purposes, in: Proceedings of the International Fall Workshop on Vision, Modeling, and Visualization (VMV'2000), Saarbrücken, Germany, 2000, pp. 231-238.

[32] D.K. Pai, K. van den Doel, D.L. James, J. Lang, J.E. Lloyd, J.L. Richmond, S.H. Yau, Scanning physical interaction behavior of 3D objects, in: Proceedings of ACM Computer Graphics and Interactive Techniques (SIGGRAPH'01), New Orleans, 2001, pp. 87-96. 